J. Comp. Int. Sci. (2015) 6(2):31-52

http://epacis.net/jicis/PDF_JCIS/JCIS-0094.pdf

jcis@epacis.net

@2015 PACIS (http://epacis.net)

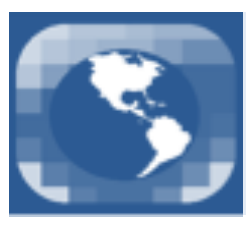

\title{
Organizing data in Astronomy: in the way for a standardized procedure
}

\author{
João Luiz Kohl Moreira ${ }^{a 1}$ and Reinaldo R. de Carvalho ${ }^{b}$ \\ ${ }^{a}$ Observatório Nacional - MCT, Rio de Janeiro, RJ, Brazil \\ ${ }^{b}$ Instituto Nacional de Pesquisas Espaciais - MCT, São José dos Campos, SP, Brazil \\ Received on Feb 25, 2015 / Accepted on August 10, 2015
}

\begin{abstract}
We discuss the world wide fast increasing mass of astronomical data and the importance of taking in account these properly storing and retrieving data procedures, since data is the most important component in the science context, understood as an empirical investigative process. A scheme of a procedure of systematically storing data is presented. We understand the need for organizing data in order to make the interaction astronomer - database easier. We introduce the framework of a universal program for storing data in a structured database, independently of the data type, provided they are in ASCII format. Guidelines to interact with this program is presented.
\end{abstract}

Keywords: astronomical databases: miscellaneous; big data; catalogs; data analysis; computational semiotics.

\section{Introduction}

The problem of data storage, which astronomers are facing more and more often, has gained an additional difficulty nowadays. Historically we observe that astronomers take care of their own problems of structuring data to be stored in data storages. Some important ports from astronomical laboratories to industry database standards are easily found in literature, like dBASE [1], from JPL and FORTH [2], from NRAO. However, dealing with huge mass of data requires specific expertise, especially after the emergence of modern paradigms like the relational database technology ([3]). Consequently we watch an increasing participation of local IT (Information Technology) staff members in decisions concerning the "modos operandis" and strategies in attacking the storing astronomical data problem, even though these engineers are not necessarily experts in astronomy.

It is now apparent that analysing scientific data no longer distinguishes itself from the filigrees of storing them. Most disturbing is that some terms registered in the vocabulary of an astronomer coincide with that of an IT-engineer but do not coincide in meaning. For instance, when a DB manager and an astronomer say "joining tables" they don't necessarily mean the same thing. The manager certainly means to join entities in the relational database sense, but the astronomer may mean to append a table to other (Figure 1). It is clear that there must be a "translation crew" linking one to another and whose ability, among others, is to understand what each part wants from the other. Furthermore, there are a number of tasks, besides reduction and analysis of data, that demand expertise about both the contents of the data and the technical aspects of accomplishing such jobs in a DB, hence requiring full-time dedication to this enterprise. This paper gathers efforts to develop a set of tools that allows DB-engineers and astronomers to talk each other easily without the peril of misunderstanding.

\footnotetext{
${ }^{1}$ Email Corresponding Author: kohl@on.br
} 


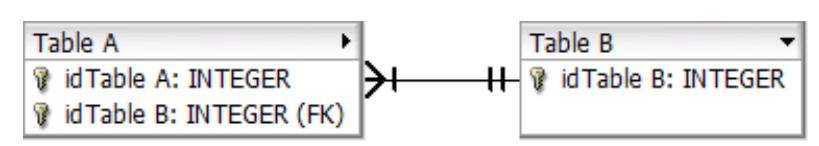

(a)

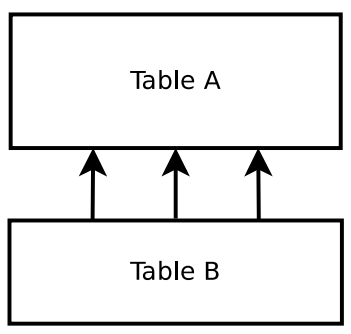

(b)

Figure 1: Differences on what a database designer and an astronomer see when they employ the term 'joining tables': (a) for a DB designer, to join two tables is to connect them under technical constraints (which details we are not discussing here); (b) for an astronomer, it may be just to append a table to the other.

Since computer sciences have emerged, they have fabulously improved the astronomical observation techniques. Software specifically conceived for astronomical purposes have been developed. Technology has grown up and astronomy has taken advantage from it. Spatial technologies have come to greatly improve astronomical observations and with them an astonishing set of software tools has been developed [4].

As a consequence of the conspicuous evolution in collecting data, astronomical data has experienced a growth in volume much more impressive than the $\mathrm{Hu}-$ man Genome Project or as huge as data from Large Harmonic Collider as seen in Fig. 1 in [5]. We could even talk about a "Moore's law" 2 for astronomical data [7].

The series of ADASS: Conferences on Astronomical Data Analysis Software and Systems annually organized since 1991 have given important ground support to this new field of astronomy. Since then, astronomers who give attention to astronomical software development can gather to talk about their last designs offering facilities to the astronomical community. Since then we witness an important improvement on the branch of astronomical data analysis, thanks to ADASS staff and associated people around the world.

Researchers from this new branch in astronomy have developed techniques that demand specific terminology and a new problem arises: how this new vernacular may fit with the old language spoken by the traditional astronomer? A sensitive point about this subject is the way astronomers see how their data are organized compared to how that data are stored in a database.

An astronomer tends to put data together according to some physical criterium, namely, he likes to put the total magnitude besides Hubble class, spectral class, redshift, morphological parameters and equatorial coordinates for a set of galaxies in a single table.

A database designer tends to put data directed in a way to make sure on its integrity at retrieving time, by doing it as fast as it gets. Data goes to different tables obeying directives connected to the domain that the related variable may have. When one wants to retrieve data, these tables are joined in order to give tuples that resemble an astronomical table, just as the astronomer expects.

This paper is aimed to introduce a scheme that links the astronomer side, given the dataset under his(her) concept, and the database designer side with his(her) integrity norms. We have two steps: assembling the structure of the database based on the entity relationship model from the data structure delivered by the astronomer, and most importantly, developing a software that will parse data from ASCII-tables and will transfer them to the database ${ }^{3}$. Nevertheless, we observe that the astronomer may change, according to his(her) needs, the way the data is organized. For instance, galaxy surface brightness may be put out of a table of photometric data and go to a specific table, because the astronomer found out additional ways of evaluating it. He wants a specific list of galaxy surface brightness coming from different methods be put side by side for each object, let us to say galaxy.

In order to set up a permanent routine to store astronomical data, at least, photometric data, we introduce a two stages procedure:

\footnotetext{
${ }^{2}$ The well known conjecture of INTEL co-founder Gordon E. Moore saying that computer's capacity doubles each 18 month [6].

${ }^{3} \mathrm{~A}$ program like this is called loading program in database professionals' jargon
} 
1. a permanent program that reads input data, whose structure is described in Section 3, and

2. schematic files, that contain a structured "meta-language" that guides the program how to read the data contained in the ASCII-tables coming from the scientific analysis procedures.

Except for maintenance purposes, the program does not need to be modified. However, the schematic files must be changed every time the structure of incoming data experiences a modification. If the data set is enriched with new kind of data, say a new classification of galaxy morphology, the database manager should think of new entities in database structure and new entries in the schematic files, but the loading program that takes information from the incoming ASCII-tables and stores it in database should remain unchanged. If the original data change only to different instances of the same kind, no changes in schematic files is needed as well.

Schematic files contain the keys for astronomical data to connect to database structure. In this sense, the schematic files are composed by a lexical that establishes the syntax for the program to read the incoming data and to store them into the database.

This way of setting up the database storage allows us to greatly improve the routine. The most important task is training users to correctly set-up the schematic files and to change them according to the variations that scientific programs may produce, leaving time for the software engineers to develop tools for other needs.

Data stored this fashion are, then, not acknowledged by the user, but by the database programmer. One must provide a mean for the astronomer to retrieve data in a context that he can easily handle it. The most straightforward way of doing it is by means of DBMS tools we call "views" [8] p. 152. Through DBMS view-tools the database manager may design views, since data are correctly stored, whose features look like the user structure of data table.

We find a three layers scheme in two directions, as seen in Fig. 2. It can be either a process of retrieving data from database and putting them in an astronomical table format, or a database loading process taking data from astronomical table and transfering them to structured database tables. To do it, one run a 'loading program' drifted by schematic files.

The schema here presented suggests a relationship with the formula E.R.C. (EXPRESSION - RELATION - CONTENT), from Semiology, introduced by Hjelmslev and discussed by Barthes [9], p. 49. According to Barthes, from semiological point of view, one has to take into account the distinction between what is the plane of EXPRESSION, where the signs are put without any signification, and the plane of CONTENT, where the signs gain the meaning that one wants them to have. For Barthes, inspired in Hjelmslev scheme, the signs to have a meaning in the plane of CONTENT, there must be a RELATION that connects it from the plane of EXPRESSION.

Taking our schema as a sample, we can see it in the context of the formula E.R.C. in two directions, one opposite to the other. First, we can understand that what is stored in a database has no specific meaning other than the strict compliance to the rules of integrity. Database manager system (DBMS) is a tool that may be useful to any application. In this sense one can get data stored in a database as being in the plane of EXPRESSION. To have a scientific meaning, one has to carry out a RELATION procedure to take data from database and to give them to the user, by having the presentation he(she) uses to recognize them as so (CONTENT). In this sense, one can see the 'views' as having the role of RELATION in the direction 'database $\rightarrow$ scientific data'.

On the other hand, data coming from astronomical presentation have no meaning for the database designer point of view, if he(she) is concerned with the database structure, namely to put them in the normal forms. For he(she) this data set is now presented in the plane of EXPRESSION. To carry data to the plane of CONTENT, under the database designer point of view, one must to run a 'loading program'. At certain point one must to have a 'dictionary' tool translating what is the input files structure (EXPRESSION for database designer) to the database structure (CONTENT). This 'dictionary' tool is understood as the RELATION. In this direction the formula EXPRESSION - RELATION CONTENT is then also applicable. The schematic files we introduce in this paper have the role of RELATION to the direction 'scientific data $\rightarrow$ database'.

Barthes ([9]) concludes that this E.R.C. ".. formula enables us to account for the metalanguages or derivative systems..." (p.49). We understand our schematic files here introduced as being a metalanguage in the context of astronomical database loading system.

The Barthes - Hjelmslev E.R.C. framework may be thought of as a template for all the Three-Tier scheme we use to find in litterature when dealing with user - database interaction [10]. 


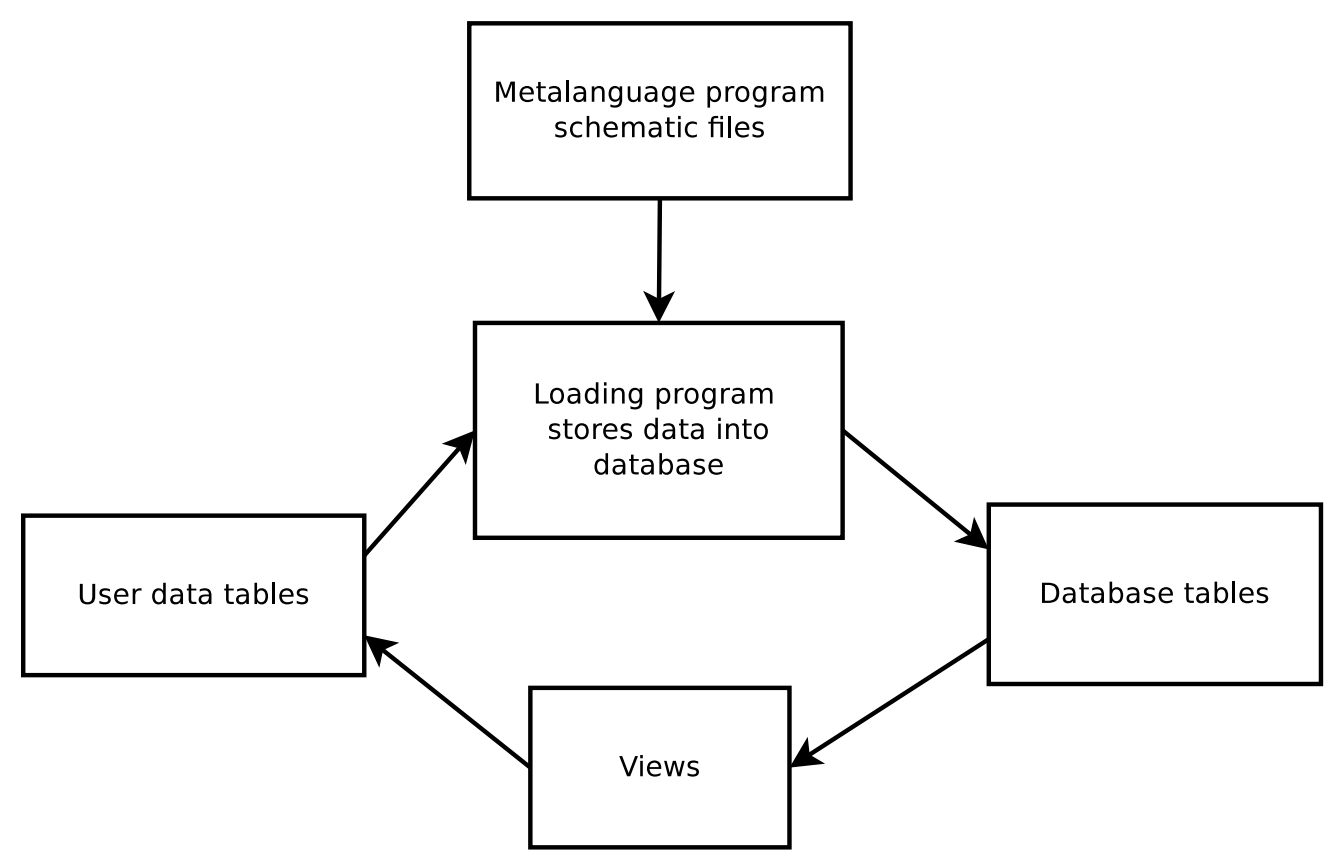

Figure 2: Three-layer scheme to store data into database from user data tables and retrieve data looking like the original tables. Both directions may be seen as different aspects of the formula E.R.C. (EXPRESSION - RELATION - CONTENT) introduced by Hjelmslev-Barthes (see text). The three cases in the center represent RELATION and the edges, the case 'User data tables' on the left and the case 'Database tables' on the right, may be EXPRESSION or CONTENT depending on the astronomer or database designer point of view. The case of schematic files may be seen as a metalanguage program.

In Section 2 we discuss two cases of photometric data coming from two different projects and the way we have pictured the schematic files to control the unique routine of storing information into databases: the SPIDER project [11] and the 2DPHOT-UKIDSS [5] project database. Both projects, based on 2DPHOT [12] calculations, are of photometric data and keep design similarities between them, although they have intrinsic particularities that should be taken into account. In this Section we also present the ER (Entity-Relationship, see [13]) models for the above cited projects, their elements, roles and cardinalities. We also present a sample of views that have been created to fit the astronomer requests in the way they want to look at their data. By querying these views and making joins on them the user may completely retrieve data in the same way their original data was organized, or, at his(her) will, to filter and rearrange coherently data in order of making his(her) science. In Section 3 we present the diagram of the interaction of the schematic files and the reading data program, which is supposed to be universal. Special attention is dedicated to the syntax of the schematic files, for they should be strictly mastered by the user. At Section 4 we discuss how the control file and the schematic file are set up and how the lexicon of metalanguage is built. At Section 5 we discuss the view, a tool that the majority of DBMS let users to costumize their queries. We present the case of SPIDER [14] as an example of this costumization. Finally, at the Conclusion, in Section 6, we present some suggestions for next database projects and some directives on how to design them to enable the astronomer interact with the system we introduce here.

Although data in both cases introduced here come as result of similar scientific analysis programs, tables of data have the same presentation as a typical table coming from any issue of photometric analysis in astronomy. So, the procedures proposed here have a high degree of universality in dealing with astronomical photometric dataset.

\section{2 - Data}

We present two cases of photometric data projects whose processes of storing information into databases were submitted to the procedures we describe here. Firstly, we have SPIDER: Spheroids Panchromatic Investigation in Different Environmental Regions [11]. 
It gathers data of bright galaxies early-type coming from SLOAN DR- 6 and UKIDSS-LAS DR- $4{ }^{4}$ and being processed by the package 2-DPHOT as described by $[11,12]$. The number of objects is fixed: 39,993 galaxies from SLOAN DR-6, 5,080 of them with data also from UKISS-LAS DR4, many of them not having information on all parameters designated to be present in their features. Au fur et à mesure that data for these objects are coming from new observations, they are being inserted into the database. This means that SPIDER is a database constantly updated. Nevertheless, this updating is not for inserting new objects, but for inserting new data for the same objects. Special care for non-existent information of objects in database should be taken into account when user is retrieving data.

Secondly we have the project UKIDSS - 2DPHOT with slightly different characteristics. Though the origin of data and the way they are processed are the same, and much of the data in SPIDER has UKIDSS data as origin [11], it is expected a huge amount of data on a possibly variable number of features. In contrast with the former project, updating an object information is unlikely, though possible. The ERmodel is simpler but its high cardinality poses a challenge when the user joins its tables.

Here comes a detailed description of these projects.

\section{1 - SPIDER}

As pointed out by [11], a set of photometric data containing 39,993 spheroidal galaxies on eight bands, four in optical range, $g, r, i, z$ from SDSS-DR6 and four in near infrared range, $Y, J, H, K$ for $5,080 \mathrm{ob}-$ jects from UKIDSS-LAS DR4 have been selected to be gathered in a homogenic database being processed by the softwares 2DPHOT [12] and STARLIGHT (see reference and discussion on how it is applied in [14]). Data may be grouped in five types.

\subsection{1 - Original data structure}

Here, the data types and quantities grouped into them, following what is described in [11]:

- ID/positioning data type:

- SDSS, RA, DEC, local ID, plate linear position, position differences according to localization method

- Photometric data type (eight bands):

- Surf Brightness, Auto and Total Mag, Kron aperture mags, color, color excess

\footnotetext{
${ }^{4}$ Details in [11].
}

- Classification and morphological data type (eight bands):

- Axes length, ellipticity, position angle, Sersic index, ellipse harmonic parameters

- Spectroscopic data type:

- Metallicity: $[\alpha / F e],[Z / H]$

- Cosmological data type:

- Redshift $(z), k$-correction

There are 295 independent quantities coming from 21 tables issued from scientific evaluation programs. Some of them, like the SDSS-ID - which is present in all tables to correctly connect each tuple to the astronomical object - does not depend on the photometric band. Others have data only in four bands, while there are those that are supposed to have information for all the eight photometric bands.

\subsection{2 - Database structure}

These sets of data are then grouped so that they will produce tables defined according to a criterion of structured relational database (see p.ex. [15]). We can see this model illustration in Fig. 3. In a central position we have the table SDSS containing the SDSS-ID and the equatorial coordinates for the EPOCH, usually 2000.0. This is a reference table being referenced by 11 relations. This means that its primary key, which is the SDSS-ID, the number of ID for the SLOAN database [11], is being used as foreign key in 11 tables. It is the one of the two reference tables with high cardinality in the database. The other primary high cardinality table is named 'SPECTS' that is described below. It remains the 11 high cardinality secondary tables which all contain scientific data. They are: Identifications, Spec_Redshifts, Mag, COORDS, Sigma, Photom_Features, Cov_Err_Spec_RMS, KC, SDSS_and_SPECTS, Metall_Feat and EBV. In addition, there are five reference primary tables containing definitions of low cardinality that qualify the instances in the data tables. They are: VelDisp_Method, EBV_Methods, Metall_Estim, Mag_Types and BANDS. Finally we have the table SPECTS, a reference table containing the spectrum name from which data were extracted. 
One expects a relationship $N: M$ between the tables SDSS and SPECTS because it is possible to get more than an astronomical object from a spectrum file as well as more than one spectrum for an astronomical object. It is well known from structured database theory that one must insert a new entity between these two ones having a $1: N$ relationship with it.

The idea behind this way of structuring the database is to take advantage of indexation methods. Minimizing the number of quantities by maximizing the number of instances allows us to improve the indexes efficiency. From the way the index is conceived, that is to say, based on B-trees [15], it is easy to see that the smaller is the number of variables, smaller is the intrication of the tree. Consequently, it is in our interest that the quantities of the same unit or covering the same domain, e.g. magnitude and surface brightness, should be stored in the same column, reducing the number of attributes in database and consequently improving its performance. To correctly qualify the instances we have to add a column to describe the meaning of the quantity instance. In order to ensure referential integrity this column usually is a foreign key referecing the name of the qualifier. All the floating point numbers are multiplied by $10^{5}$. This factor is the limit of accuracy of observations and doing this we can have much more celerity using indexes to perform searches.

Below we describe the tables and their quantities inside the SPIDER database as they were spanned according to the entity relationship model showed in the Fig. 3. Additional details about the quantities can be found in [11].

updates: A table called updates just stores information about the time data was inserted and updated. It has no relationship with the other tables. It contains the keys:

idupdates: primary key, an autoincremented integer key;

date: date \& timestamp date;

comments: notes or comments that eventually are appropriate.

In the center of the diagram of Fig. 3 we find two reference tables to which all the secondary tables are related. They are:

SDSS: This table contains the SLOAN catalog number of identification and it is used as primary key to reference all the quantities pertaining to an object. It has the keys:

idSDSS: The SLOAN catalog number, primary key;

$R A, D E C$ : Equatorial coordinates in degrees and decimal fraction;

EPOCH: The epoch used to take the coordinates (usually 2000.0).

BANDS: This table contains the name of the photometric bands used in the database data. The keys are:

idBANDS: An autoincremented value, used as primary key;

name: Usually a character defining the band, nevertheless possibly containing more characters describing it.

Usually the data tables have the keys

idSDSS : Foreign key referenced to the table SDSS, and

idBANDS: Foreign key referenced to the table BANDS,

but tables containing data extracted from spectra reduction do not contain the key idBANDS. Consequently these keys are not being described below. Here are the tables defined inside the database and the quantities they contain:

Photom_Features: as 'Photometric Features', this table contains the photometric parameters for the objects mainly deduced from the software S-Extractor (see details in [12]). Their quantities are:

a4: The cossine coefficient in the Fourier series galaxy isophote fitting;

sep: difference in pixels between the expected position of the center of the galaxy and the position found by 2DPHOT;

mi_e: mean surface brightness;

re_e: effective radius;

$b a$ : axis ratio of the fitted ellipse;

pa: position angle of the ellipse major axis;

sn: Sersic index;

seeing: adopted seeing assumed to be convoluted with the galaxy image; 


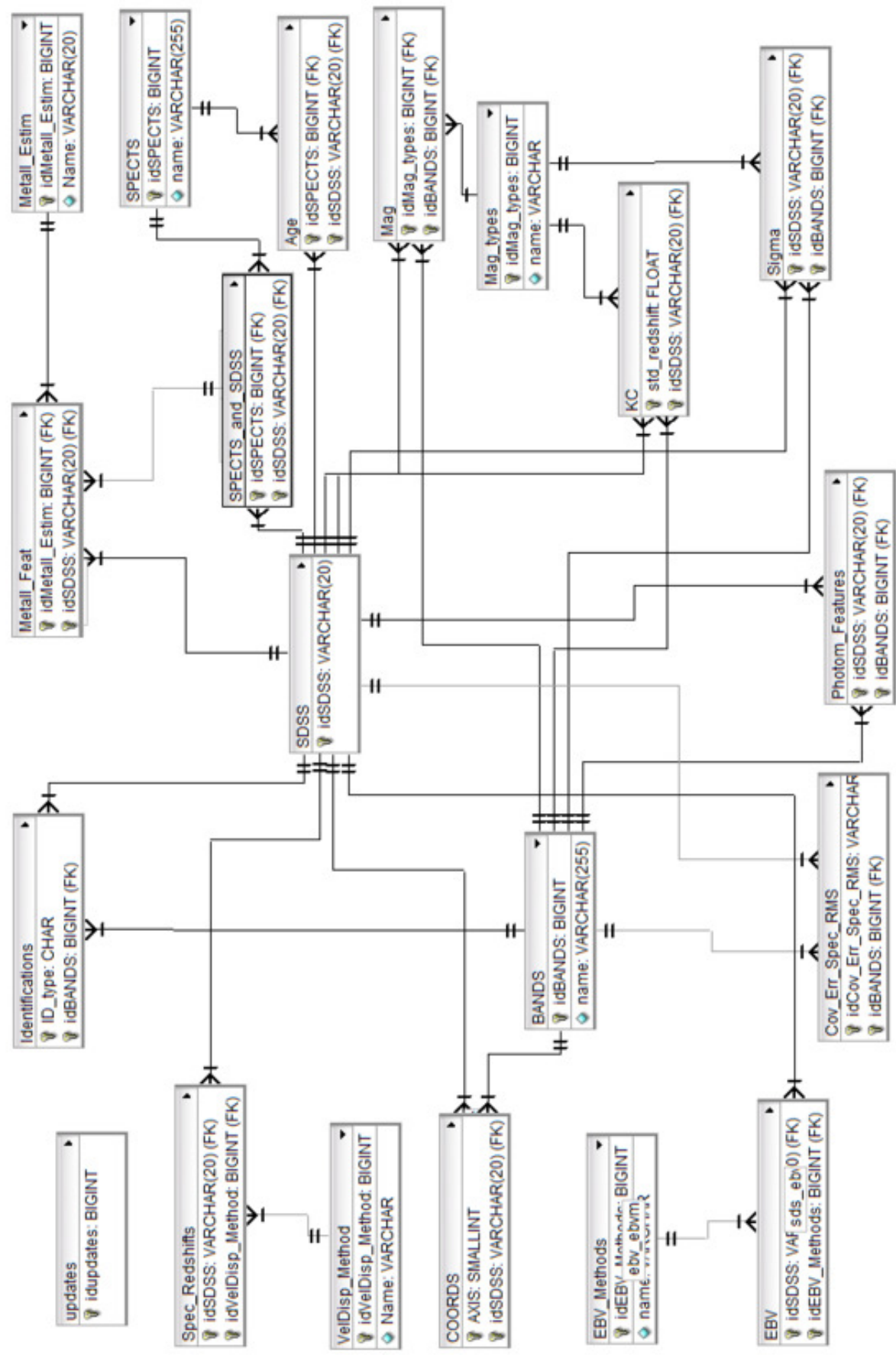

Figure 3: E.R.-model for SPIDER database. The diagram in this figure and in Fig. 4 were designed with the aid of the software DBdesigner FORK (http://sourceforge.net/projects/dbdesigner-fork/). Entities showed here are in the reduced form. 
scale: adopted image scale;

$A_{-}$Extinction: extinction correction.

VelDisp_Method: as 'Velocity Dispersion Method', reference table that contains the names of the different methods used to estimate velocity dispersion. Their keys are:

idVelDisp_Method: an autoincremented value, used as primary key;

Name: It contains the names of the velocity dispersion methods.

Spec_Redshifts: as 'Spectral Redshifts', this table contains the spectrum derived values relating the redshift and dispersion velocity. It has:

idVelDisp_Method: Foreign key referenced to the reference table VelDisp_Method;

$z$ : Redshift;

VelDisp: Velocity dispersion;

VelDisp_sigma: Standard error in determining veldisp.

EBV_Methods: which means 'Method of finding Excess on colour index $(B-V)^{\prime}$, in magnitude. Reference table, it contains the names of different color excess computed by 2DPHOT. Their keys are:

idEBV_Methods: An autoincremented value, used as primary key;

name: The different names of the color indexes.

EBV : It contains the values of color indexes of the objects. Their quantities are:

idEBV_Methods: Foreign key referenced to the table EBV_Methods;

value: The value of the index for the referenced EBV method.

SPECTS: this reference table contains the names of the spectra from SDSS used to the determination of spectral quantities. This table is left to eventually have additonal instances in the future, in case it is necessary. Apparently, from SLOAN-DR6 homepage, there is a $1: N$ relationship between the objects and spectra. Nevertheless, it could be possible that this situation may change, so, we have decided to define a $N: M$ relationship between these two tables, giving rise to an additional table gathering information from both. When we have two tables in $N: M$ relationship we create a third table that allows us to disentangle it, so that this new table has a $1: N$ relationship with each of these original tables. This additional table is described at the next item. For while SPECTS has just one key, it may contain more, according to the need.

idSPECTS: The name of the spectra as defined from the SLOAN catalog.

SDSS_and_SPECTS: This is a table designated to disentangle the $N: M$ relationship between tables SDSS and SPECTS. It has the combination of the foreign keys idSDSS and idSPECTS as primary key, so it has with both tables SDSS and SPECTS a $1: N$ relationship each (Fig. 3, near the up-right corner).

idSDSS: Foreign key referenced to the table SDSS;

idSPECTS: Foreign key referenced to the table SPECTS;

Age: Value of the measured age. It depends exclusively from the object defined in table SDSS and the spectrum defined in table SPECTS.

Metall_Estim: as 'Metallicity Estimation', reference table containing the name of the quantities used to trace metallicity, like $[\mathbf{Z} / \mathbf{H}]$ or $[\alpha / \mathbf{F e}]$. Their quantities are:

idMetall_Estim: An autoincremented value, used as primary key;

Name: The name of the quantity.

Metall_Feat: as 'Metallicity Features', this table contains informations about the metallicity of the galaxies in database. Their quantities are:

idMetall_Estim: Foreign key referenced to the table Metall_Estim;

$i d S P E C T S$ \& $i d S D S S$ : Foreign keys referenced to the tables SPECTS and SDSS;

val: value of the metallicity. 
Mag_types: as 'Magnitude Types', reference table, contains different magnitude type determination names. It does not contain photometric bands which have their own table differentiation (BANDS). This table here is to take account of the way the quantity magnitude was obtained, different apertures, even surface brightness. Their keys are:

idMag_types: An autoincremented value, used as primary key;

name: The name of the method.

Mag: Table holding values that concerns the magnitude values on the database objects. Their keys are:

idMag_types: Foreign key referenced to the table Mag_types;

value: value of the magnitude.

KC: as 'K-Correction', this secondary table holds all values of K-corrections. Their quantities are:

std_redshift: the value of the redshift used to evaluate the K-correction. Presently the value may be 0 or 0.0725 ;

idMag_types: Foreign key referenced to the table Mag_types;

value: the value of $\mathrm{K}$-correction.

identifications: Secondary table. This table holds all kind of identifications present in the observed data, like the S-extractor catalog number and UKIDSS/SDSS catalog number. The quantities are:

id_type: A character defining the kind of identification;

value: quantity containing the ID value.

COORDS: Secondary table, it contains data related to coordinates of object in the frame of origin. Their quantities are:

axis: May be 1, if the tuple concernes the coordinate $x ; 2$, if it concernes to coordinate $y$;

value: The value of the coordinate.
Cov_Err_Spec_RMS: as 'Covariance Error Spctrum RMS', secondary table, contains computed errors on optical photometric effective parameters as a function of the logarithm of the $\mathrm{S} / \mathrm{N}$ per pixel (details in [11]) and the $\chi^{2}$ obtained from two-dimensional fitting and the modeling of PSF. Their quantities are:

idCov_Err_Spec_RMS: Key specifying one of the errors;

value: The error related to the key.

Schematic files for storing data into the database by means of loading program are discussed in Section 4.

\section{2 - 2DPHOT - UKIDSS}

Contrary to the previous case, although originated from the same sources and processing package, 2DPHOT-UKIDSS database is neither about a specific class of objects nor limited to any amount. It is built to house a large amount of data, which increase continuously.

\subsection{1 - Original Data Structure}

It has only three of the five data type SPIDER has, as described in Section 2.1.1. According to the 2DPHOT readme file, data in file 'big_cat.dat' may be grouped in the following types:

- ID/Positioning data:

- ID, XIMA, YIMA, RA, DEC, FWHM, respectively, the ID from S-Extractor processing, central plate position of the object, equatorial coordinates and the estimated Full Width Half Maximum for the object image;

- Photometric Data:

- KRAD, MAUTO, erMAUTO, $\operatorname{MAP} n$, $\operatorname{erMAP} n$, ¡mi $i_{-} \mathrm{e}$ and MT, respectively, the Kron radius, S-Extractor AUTO_MAG, aperture mags $(n=1 \ldots 27)$ and their estimation error, surface brightness inside the effective radius, and Total Magnitudes and their estimation error;

- Classification / Morphological data:

- 2DFLAG, S/G, FLAG, all classification linked to stellarity index (S-Extractor), 'r_e,2D' effective radius and ellipse harmonic parameters and the error estimators. 
It totalizes 133 independent values coming from just one ASCII file. They are, then, re-organized to fit the database structure that we describe below, in Section 2.2.2.

\subsection{2 - Database Structure}

The E.R.-model for these data is shown in Fig. 4. Compared to the SPIDER database structure, this one looks like simpler, as consequence of having less types of data. "Processing" is of central importance in this database structure, since the data comes mainly from S-Extractor running over a dataset for the frame of a sky field. Each process defines a

Like in the SPIDER case, low cardinality tables contains information about methods used to evaluate the different instances of resulting data. They are:

TrigoMethods: may be the letters 'R', 'B', 'm' and 'M', respectively refering to the determination method for the terms of Fourier decomposition of the isophotes $\left(a_{3}, b_{3}\right.$ to $\left.a_{5}, b_{5}\right)$, from the zero-order ellipse fitting, according to Bender method, median along profile and at the peak ${ }^{5}$;

PhotoMode: may be: "Mean Surface Brightness", "Central Surface Brightness", "MAG AUTO" and "MAG TOTAL";

PhotoMethods: effective radius method determination; may be: "S-Extractor", "2DFIT", "GROWTH", "2D Sersic", "2D Seeing Sersic" and "1D Sersic".

We have the high cardinality tables that contain all the information about the scientific and analysed data:

SexCat: information coming from running the program S-Extractor, except some features which are also obtained from programs running methods. The latter go to other high cardinality tables, described below. To know more about S-Extractor resulting features see S-Extractor documentation, namely [16];

TrigoData: galaxies from table SexCat and their Fourier terms, $a_{3}, b_{3}$ to $a_{5}, b_{5}$ for the different methods of determination. Their features are:

Param: A 2-character key having values of Fourier coefficients;

\footnotetext{
${ }^{5}$ refer to $2 \mathrm{DPHOT}$ documentation for details.
}

dataset. In addition to S-Extractor, other features determination methods may be applied, like TrigoData, which is evaluated by the 2DPHOT own method; PhotoData, EffectiveRadius and so on, all part of 2DPHOT package (see [12]) . "Bands", storing information about photometric bands, is also a primary table in this scheme and connects to the table "Processing" in a 1:N relationship basis. By its turn, "Processing" "has "SexCat" in an 1:N relationship, too, because one can run 2DPHOT on several frames in a same process, provided one has the same band for all of them. "SexCat" connects as primary table for six data tables.

idTrigoMethods: foreign key refering to the table TrigoMethods, described above;

idsexcat, idprocessing: foreign key, refering to the table SexCat.

PhotoData: galaxies from table SexCat having some of photometric calculations like:

ellipticity: the ellipticity parameter, being $1-$ $b / a$, where $a$ and $b$ are, respectively, the major and minor axes of the fitted ellipse;

$\boldsymbol{p a}$ : position angle, the major axis angle relative to the horizontal axis;

sersicindex: the $n_{e}$ Sersic parameter;

$r m s$ : the Root Mean Square value of the fitting process, according to adopted method.

EffectiveRadius: galaxies from table SexCat having the $r_{e}$ from different methods of evaluation. Some of these methods are not present in attributes like ellipticity and $p a$, so these ones will have be attached with value null.

PhotoMags: different magnitude method evaluations, having only the feature value.

apertureMags: apperture magnitudes from SExtractor (see S-Extractor documentation) and their uncertainties:

apertureindex: index of aperture given by SExtractor;

value: the value of concerned magnitude uncertainty: its uncertainty. 


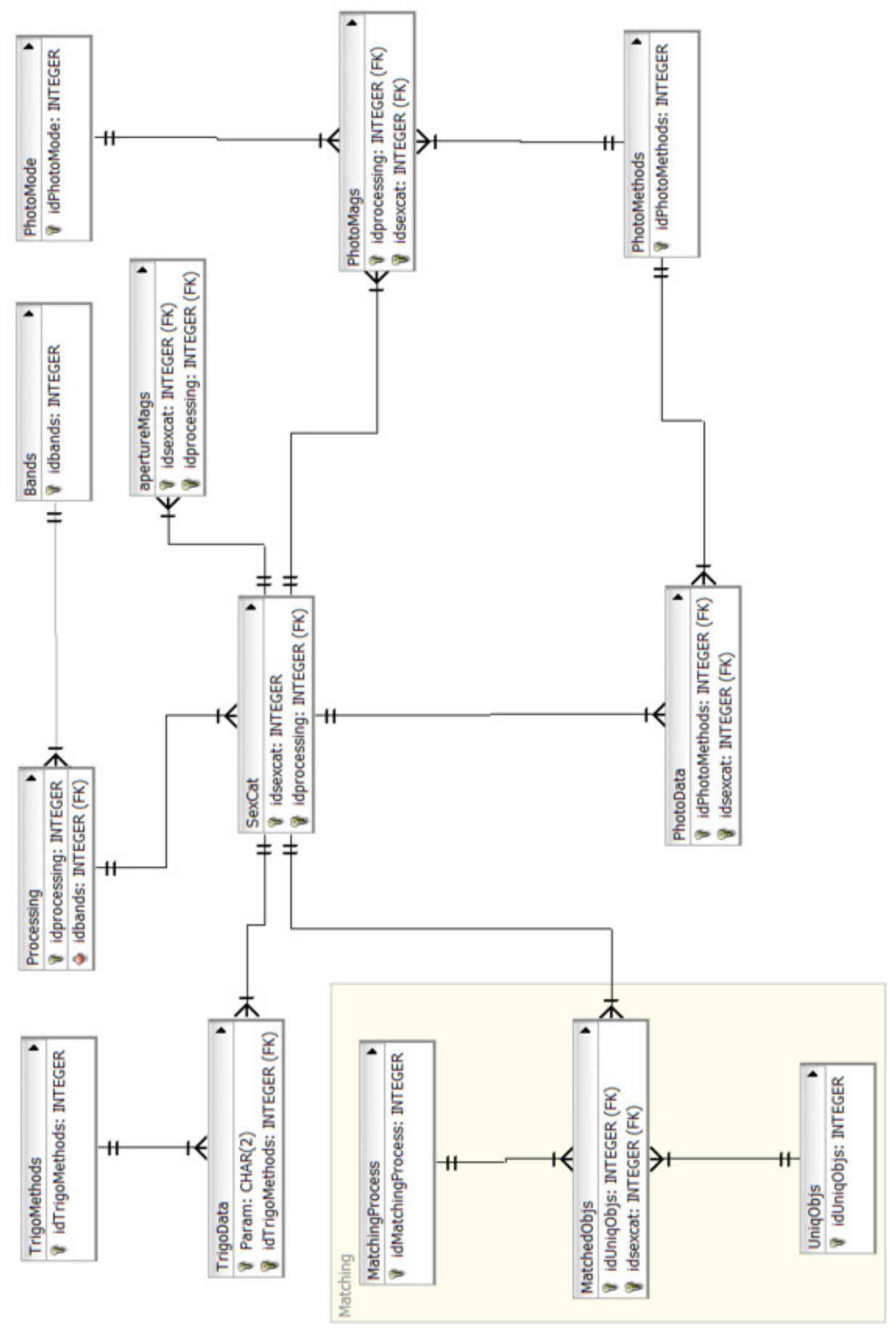

Figure 4: E.R.-model for UKIDSS database. 
Additionally, there is a section specially dedicated to the matching process. Since data is being inserted into the database, sky frames overlapping themselves, for the same, and for different photometric bands should be matched, otherwise it will be an important handicap in the database. Nevertheless, matching process is far from be standartized $([17,18])$, so astronomers and administrators should be free to perform as many matching procedures on overlapping fields in the database as they find appropriate. If, initially one could conceive the database being built on unique astronomical objects, it shows up not to be the most suitable decision, for the tools to determine object unicity are not well established in the astronomical comunity.

There is a section in the lower-left corner of Fig. 4 where three entities are dedicated to store information about the matching processes performed on the data in 2DPHOT-UKIDSS database. The first table is that identifies the matching procedure, called

MatchingProcess: it has the columns:

idMatchingProcess: primary key, a self incremental number that allows the table to point to a sole matching process;

Name: the name of matching process;

Comments: notes from administrator if appropriate;

Date: The execution date.

The second table is

UniqObjs: that contains just one column, a self incremental number. It will be used to identify matching objects from different frames of overlapping fields. One could argue that it should not make sense to maintain a one-column-table. However, this instance is necessary when we think that, to have a real structured database, one should store the unique identifications before any other feature in the database. But, in our case, the unique identification is, by its nature, an a posteriori process, furthermore, not being an ultimate procedure.

The third table is

MatchedObjs: which has:

idsexcat, idprocessing: object ID (from table SexCat);

idUniqObjs: foreign key, referencing table "UniqObjs";
idMatchingProcess: foreign key, referencing table "MatchingProcess".

In Section 4 we discuss the schematic files for storing data into the database using the loading program which is discussed below, in next Section.

Database views may be designed to reproduce the original data structure, as presented in the file 'big_cat.dat', from original data set. Views for 2DPHOT-UKIDSS database are discussed in Section 5.

\section{3 - A (nearly) Universal Data Storage Rou- tine}

We have developed a program in JAVA to read the data files, led by schematic files describing the way input data were built, and how to store these data into database, whose structure is also described by these schematic files. The program connects with the database by means of the default database connectivity (JDBC). In the present case we use the PostgreSQL manager system (DBMS) to store data. Then, the program interprets the schematic files that points out where to take data, stored in ASCII-table format and how to re-organize them such that they may be put in the database tables according to the structure these tables were conceived (Fig. 5). The schematic files then play a fundamental role in the data-transfer process. The way they are written should be strictly tuned with both the database structure and the ASCII-table organization.

The program is divided in the following modules:

1. Connection to the database;

2. Getting information where input data come from;

3. Getting rules to read the input data;

4. Getting rules on how to put data into the database structure and

5. store this data according to this structure.

Two additional modules should be put:

6. Spelling the schematic files and scanning them for the sake of correctness and consistency of the rules;

7. Scanning input data files for the sake of correctness and consistency of data. 


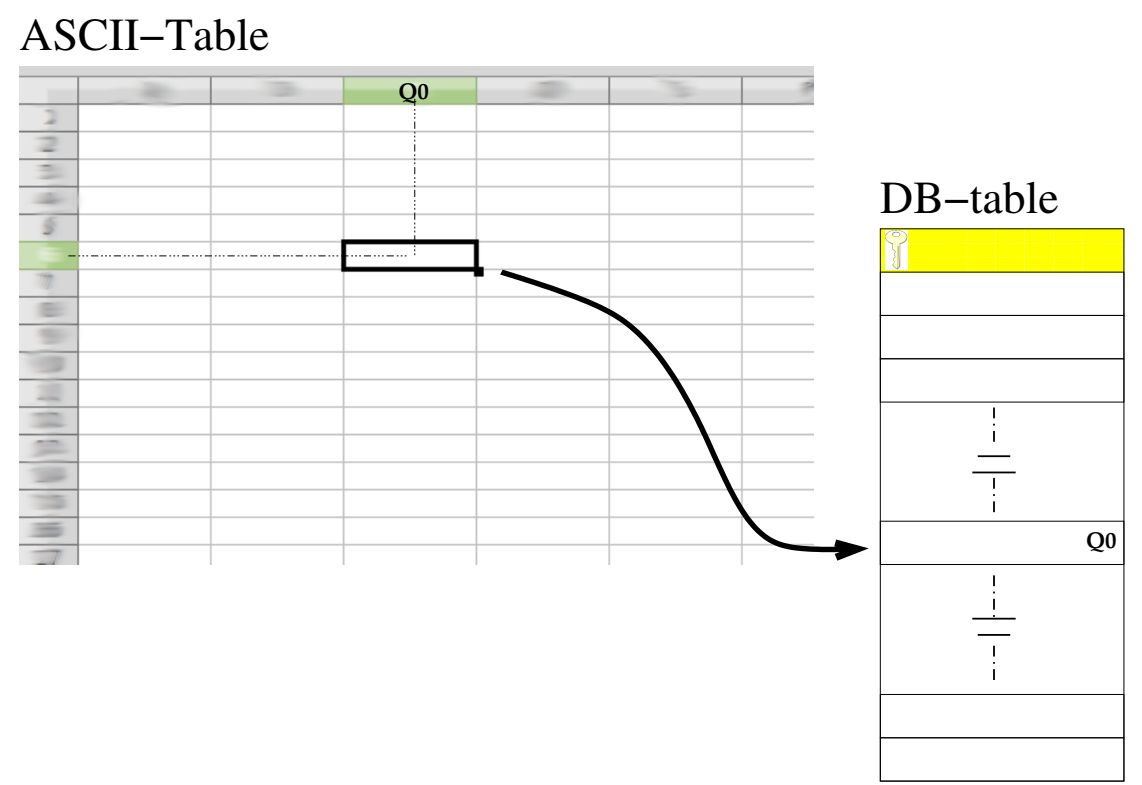

Figure 5: Transferring data from an ASCII-table to a DB-table. This scheme is supposed to be done by the loading program, led by the schematic files.

The two last points are very sensitive, since any misspelling or inconsistency in a schematic file or data file may make the database untrustworthy and may even make its use inviable.

Directives to link data from ASCII-table to DBtable are set up in a straightforward fashion, something like: data in the ASCII-table column number ' $\mathrm{X}$ ' should go to a given column in the DB-table, primary key and foreign keys properly defined. Nevertheless some degree of shortcut and deductive definitions are desirable, otherwise we risk of having to write too large schematic files, increasing chances of misspelling and other kind of errors on doing it. Under the astronomer/custom cooperation it may be possible to make generalizations for statements to span over a same class of data. For instance, it may be possible to define names of columns: the program is able to read the first row in an ASCII-table and interpret it as a "header" for columns in the rows coming below it, just as a worksheet CSV-file with columns separated by blank spaces and lines separated by LF characters. Hence, we can define a class of columns, whose names obey a pattern. Then, we can attach these columns to a quantity or a group of instances pertaining to the same quantity in the database. For instance, the quantity a 4 and its values for different bands may be named as a4_g, a4_r and so for. Instead of writing a statement for each band saying that $\mathrm{a} 4_{-} \mathrm{g}$ is to be stored in the column $a 4$ of Photom_Features DB-table having the foreign key $\boldsymbol{i d b a n d}$ set to 'g', and so on, we may define a pat- tern "a4". Therefore we can state that everything begining with a4 is to be stored in the column $a_{4}$ in DB-table Photom_Features. Procedures defining rules of extracting the photometric band value from the end of the column name are, then, introduced. With the same thought, a set of operator symbols are introduced to provide the program with ways of spanning sequences of column numbers or file names. Functions performing string operations and numeric calculations may be defined in the schematic files, too.

A better idea on how this system works may be seen in the way the schematic files are set up, presented below in Section 4.

After taking the rules of reading ASCII-tables and storing data in the proper columns of DB-tables the program begins to read data and organize them in order to build blocks of information to be transfered to the database. It is, anyway, a laborious process, specially if rows in ASCII-tables are shuffled in the sense that each table contains a different object ID order. From experience, we see that the more ID order are equivalent in ASCII-tables, the faster the loading process. Otherwise, the program that runs in a finite memory computer, needs to paginate data into disk to deal with the amount of data it has to stream.

\section{4 - Schematic Files: the Control File and Data Structure File}

The schematic files are written in compliance to 
the YAML $^{6}$ standards. They seem to be more compact and human readable than the format XML, which would likely need for a GUI tool to be built, unlike YAML that needs only a simple text editor, like Emacs or vi. On the other hand it is preferable to adopt a format ready and already widespread in the public domain than to invent a new one. We are looking for a way of parsing instructions to a program not in a form of algorithm, but in some kind of serialized information. YAML is a project turned to data serialization which is what we are interested in.

There are two kinds of schematic files: the control file and the data structure file. The former is a set of statements that contains metadata as information necessary to establish the database connectivity, the name of database, the ASCII files where data come from, some rules for numeric transformations and the set of reference DB-tables, i.e. the low cardinality relations. The latter file contains specifications on how to get data from ASCII files and store them into the database tables.

\section{1 - Control File and Structure File for SPI- DER}

Samples of schematic files for storing SPIDER data into database is shown in Fig. 6. In the illustration of the ControlFile we see the keywords: 'database:', 'bands:', 'ebv_methods:', and so on, each followed by rows that define fields concerning these keyword specifications. Characters are put in green or blue by the text editor used that recognizes automatically the format YAML. The keyword 'database:' is mandatory, since their fields establish connectivity settings, the name of the database (SPIDER) and other essential definitions.

A field specification is in the form of

[name of the field]: [definition]

Where [name of the field] is self explanatorily, and [definition] is a set of statements that are necessary to adequately define what is going to be stored under the given circumstances about this field. Each line defines a field.

\subsection{1 - The Control File for SPIDER}

For the keyword 'database:' the mandatory fields are:

driver: related to the JDBC connection driver;

connectivity: the driver path and file name, according to JDBC syntax specification;

\footnotetext{
${ }^{6}$ http://www.yaml.org/spec/1.2/spec.html
}

engine: the database name;

user \& password: the username and password for the DBMS controlling program;

path: the name of the folder where the ASCII table files are kept. The value 'null' means these files are in the current folder;

file: the list of ASCII files where data is put in, separated by a ';';

columns: list number of columns in the ASCII table files in the same sequence as the list in the field 'file';

lines: number of lines of each table file in the same sequence as columns;

datastructure: the name of the data structure specification file;

blocklines: the number of lines read at once from ASCII files and processed to be stored into database. Small number means processing delaying, because the program will be forced to read from disk more times; a too big number affect the RAM memory occupation and may force the system to paginate on disk, also delaying the processing. We have to find a number that balance these two limiting conditions. Typical value for blocklines is 1,000 .

nullvalue: this keyword defines the ASCII code for an undefined value. Scientific analysis programs are usually designed by the astronomers themselves. They use to define a discrepant number to tag a result as undetermined, when this is the case. The package 2DPHOT, is set to tag the value 999 or 99 for these cases. In database standards we use to set the value NULL when an instance is found to be undefined. The keyword 'nullvalue' is just to warn the loading program that the value, namely, 999 or 99 , is to be replaced by the value NULL to be stored in DB-table. This transformation is necessary to not corrupt the indexation procedure.

Items of lists are separated by a semicolon. A keyword beginning with the character underscore ("_") is taken as an operator definition. The parameter for the operation is set to " $\mathrm{x}$ " by default. Keywords beginning with '\&' are understood as operators. In the ControlFile seen in the Fig. 6 we see the definition of 
the operator_normalize as being the integer part of the result of multiplying the operand by $10^{5}$.

Operators are the set of internal functions of package 'Math' in JAVA and the arithmetic operators are defined as functions: \&sum, \&subtract, \&multiply, \&divide and further simple functions like \&integer, \&round and \&frac, all they selfexplanatory.

Following the YAML specification, keywords are separated by a line containing "---!" followed by comments. Fields of keywords are tabbed by a blank character. Some characters should be escaped by the backslash sign (")"), namely ampersand ("\\&") and comma (" \,").

Coming next in Controlfile is the definition of the reference low cardinality DB-tables. These tables contain data that are not coming from the ASCII data tables. The data in these DB-tables are used to qualify the analysed data in order to correctly reflect the scientific qualities of the data. For instance, the entity bands, as determined by the keyword attributes, coming below, contains the instances 'g', ' $r$ ', ' $i$ ', ' $z$ ' etc, that qualify some quantities like magnitude with the observing photometric band related filter. 'ebv_methods' contains $\mathrm{E}(\mathrm{B}-\mathrm{V})$ and so on, that differentiates the color excess values, according the SPI-

reference: composed by quantities definition in the form ' $[k e y]=[v a l u e] '$. Each key represents a quantity in the database table being referenced. For instance, the keyword reference for 'photom_features' contains the definition 'bands=\&substring (\$idss . indata $\backslash, 6 \backslash, 7)$ '. The key 'bands' means that the table 'photom_features' has a reference to the table 'bands' and its value (in the referenced table) is determined by a specific value, in this case, a substring composed by the positions 6 included until 7 excluded (it means just the position 6) of the field defined in the keyword 'idss', qualifier 'indata', which comes below;

name of keyword: all of the following fields for a keyword represent quantities in the related database table. The name of the quantity is separated from its definition by a colon. The definition is composed by qualifiers that are defined in the form '[qualifier]=[value]'. The qualifier definitions are separated by semicolons. The first qualifier should be 'indata $=$ [pattern of filename]'. It points
DER Readme file. It is in this ControlFile that we introduce the table definition and its instances for entities like Metall_Estim, VelDisp_Method and Mag_Types, all of them described in SPIDER Readme file.

\subsection{2 - The Data Structure File for SPIDER}

In the lower part of Fig. 6 we can see an extract of the file 'datastructure' used to store original data of SPIDER project. Each keyword is the name of database table used to store astronomical data: 'sdss', 'spects', 'photom_features' are examples of these tables. Compare these names with the items presented in Section 2.1.2. Immediately below each keyword definition we see the field 'reference'. This field is mandatory for all the keywords defined in this type of file. It generally contains the definition of foreign keys for the instances defined in the field. It also may contain a static definition, like for the keyword 'sdss', the definition of 'epoch=2000'. It means that the quantity 'epoch' in the table 'sdss' is to be statically set to the value of 2000 for every instance. The other fields contain the name of the quantities in the table and the way the ASCII tables should be accessed and the columns they should come from. The syntax for these field definitions is summarized below:

out the ASCII file to be read. Usually the pattern definition follows the "regex" standards found in the UNIX-like systems and derived tools such as languages like PERL or JAVA. For instance, in the 'indata' qualifier definition for 'photom_features' in Fig. 6, we see the value 'struc_._table'. It means that the input ASCII tables should be every file beginning with 'struc,', followed by whatever else, and then finishes with '-table'. After that, it comes 'colnumber 1 ' and 'colname=SDSS_ID'. The entire definition means that it is to read all files beginning with 'struc_'t something +' 'table' and take the first column, that has the name 'SDSS_ID' and store the data coming below at the database table 'sdss', in the column 'idsdss'. Analogous procedure is done for the other keywords, 'sep', 'mi_e' etc. In these cases there is one more qualifier field, for instance under the keyword 'sep', we have '\&normalize(\$sep.colvalue)'. Words beginning by '\&' are understood as operators. In this case, the operator 'normalize' was defined in the Control File, but it could be any recognized 


\section{ControlFile}

\section{-.. ISPIDER \\ database: \\ driver: org.postgresql.Driver}

connectivity: jdbc: postgresql://localhost/

engine: SPIDER

user: postgres

password: postgres

path: null

file: a4 table.dat; coordinates table.dat; ...

columns: $7 ; 27$;

lines: 39993.39993 .

datastructure: Datastructure

datastructure: Data

blocklines: 1000
nullvalue: 999.0

nullvalue: 999.0
normalize: (\&integer(\&multiply $(x \backslash, 100000.0)$ )

-.- ISPIDER

bands:

attributes: name $=g \backslash, r \backslash, i \backslash, Z \backslash, Y, J, H \backslash, K$

... ISPIDER

ebv methods:

at $\bar{t}$ ributes: name $=E(B-V) \backslash$, Corrected $E(B-V)$

... ISPIDER

-.. !SPIDER

DataStructure

sdss:

reference: epoch $=2000$

idsdss: indat $a=\wedge$ coordinates; colnumber $=1$; colname $=$ SDSS ID

ra: indata $=\wedge$ coordinates; colnumber $=2 ;$ colname $=\wedge$ RA; value $=$ $=$ nnormalize $(\$ r a$. colvalue $)$

dec : indata $=\wedge$ coordinates; colnumber $=3 ;$ colname $=\wedge D E C ;$ value $=$ \&normalize ( $\$ d e c$. colvalue)

... ! SPIDER

spects:

reference:

idspects: indata=^DR6 ETGs SL alphamiles SPpar; colnumber=2; colname=IDspec

-.. !SPIDER

coords:

reference: bands $=\$$ substring ( $\$$ value . colname $\backslash, 2 \backslash, 3)$; axis $=0$

idsdss: indat $a=^{\wedge}$ coordinates; colnumber $=1$; colname $=$ SDSS ID

value: indata $={ }^{\wedge}$ coordinates; $\operatorname{colnumber}=4-18-2 ; \operatorname{colname}=\bar{\wedge} x ;$ value $=\&$ normalize $(\$$ value. colvalue $)$

-.. !SPIDER

coords:

reference: bands $=\$$ substring ( $\$$ value, colname $\backslash, 2 \backslash, 3)$; axis $=1$

idsdss: indat $a=^{\wedge}$ coordinates; colnumber $=1$; colname $=S D S S$ ID

value: indata $=\wedge$ coordinates; $\operatorname{colnumber}=5-19-2 ; \operatorname{colname}=\wedge y ;$ value $=£$ normalize ( $\$$ value. colvalue)

...

mag:

reference: bands $=\&$ substring (\$value. $\operatorname{colname} \backslash, 5 \backslash, 6) ;$ idmag types=\$substring $(\$$ value. colname $\backslash, 3 \backslash, 4)$

idsdss: indata $={ }^{\wedge}$ mag $k$ ron $i$ table; colnumber $=1$; colname $=$ SDSS ID

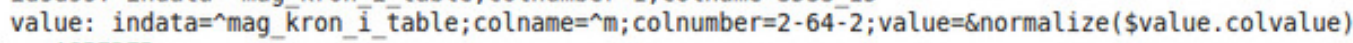

-.. !SPIDER

photom features:

reference: bands=\&substring ( $\$$ idsdss. indata $\backslash, 6 \backslash, 7)$

idsdss: indata $=^{\wedge}$ struc. table; colnumber=1; colname=SDSS ID

sep: indata $=^{\wedge}$ struc. table; colnumber $=2 ;$ colname $=\wedge$ SEP; value $=$ \&normalize ( $\$$ sep. colvalue)

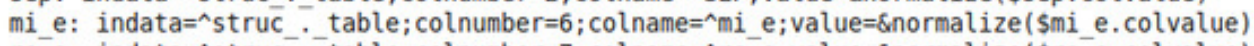

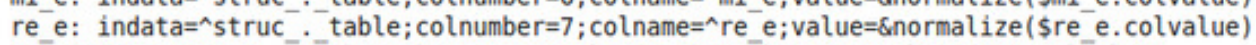

ba: indata $=\wedge$ struc . table; colnumber $=8 ;$ colname $=\wedge b a ;$ value $=\$$ normalize $(\$ b a$. colvalue $)$

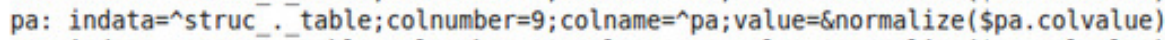

sn: indata $=\wedge$ struc ${ }^{-}$. table; colnumber $=10 ;$ colname $=\wedge n ;$ value $=\&$ normalize ( $\$$ sn. colvalue)

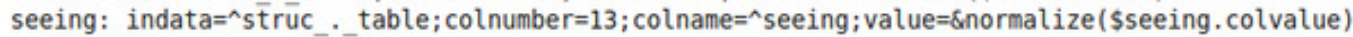

Figure 6: Samples of schematic control file and data structure file contents. 
function defined in Java language. It opearates on '\$sep.colvalue'. Words beginning with '\$' are understood as keyword name. 'colvalue' means the values that are parsed from the columns in ASCII table to be put in the column named 'sep' in DB-table. The result of these statements is: in the column named 'sep' in DB-table photom_features will be stored values coming from all columns whose name begin with the word 'SEP' from the ASCII filename 'struc $+[$ a letter supposed to be the photometric band name] +_table' after being multiplied by $10^{5}$ and the getting the resulting integer part. For each instance stored in database it will be stored in the foreign key 'idbands' referencing to the primary table bands whose value is to be taken from the character 6 of the ASCII table filename ${ }^{7}$.

Notice in the field specification of the attribute 'value' for the keyword 'mag' we have 'colnumber=2-64-2'. This is definition of kind ' $\mathrm{N}-\mathrm{M}-\mathrm{K}$ ' where ' $\mathrm{N}$ ' is the first numerical (integer), ' $\mathrm{M}$ ' is the last one and ' $\mathrm{K}$ ' is the step means that the columns of number 2 to 64 , taken 2 by 2 of the given file (all files beginning with the pattern 'mag_kron_i_table') is to be considered to be stored in the attribute 'value' of DB-table 'mag'. This statement is similar to a loop definition in FORTRAN-77: 'DO x $\mathrm{J}=2,64,2$ ', meaning that the block of instructions coming below until the first line 'END DO' should be repeated and the variable 'J' should take the values $2,4, \ldots 64$. If the step is omitted, as in FORTRAN, it is assumed to be the unit.

\section{2 - Control File and Structure File for 2DPHOT - UKIDSS}

The model of entity relationship for database UKIDSS is shown in Fig. 4. As we have seen, these data have a slightly different architecture, although coming from the same processing procedure. We can see that the model is simpler, compared to the SPIDER data strucuture. In either case the data loading process is by any means neither faster nor simpler.

\subsubsection{The Control File for 2DPHOT - UKIDSS}

Similarly to the SPIDER case, Controlfile contains information for database connectivity and description of the low-cardinality DB-tables. Notice that there is only one ASCII data file: 'big_cat.dat', as pointed out in the README file of 2DPHOT distribution package. This file is supposed to contain all information relevant to astronomical applications.

The low-cardinality tables are 'bands', which, contrarily to the SPIDER case, there is no way of retrieving photometric band information from the input ASCII data. This information should be supplied by the administrator for the specification of the sky field under study. Here, for the sake of illustration, the band is ' $r$ '; 'photomode', that defines the kind of magnitude being stored; 'photomethods', that defines the methods used to determine photometric data (details in [12]); 'trigomethods', the methods applied to determine the parameters of the Fourier series expansion of the fitted ellipses in galaxy images; and 'processing', that defines information on the loading process. In any field specification we can put the value "<localtime $>$ " that determines that it is to be replaced by the local time in the instant the process takes place. Usually this value is inserted in the attribute 'date' of the field defining the table 'processing'. The keyword "save", that we can see in the field specification "references:", is necessary because the table "processing" is a primary table itself, although it contains a reference to the table "bands". This field states that it is necessary "to save" its own primary key "idprocessing" during the processing run time to be used by the data tables. This key is being referenced in the high cardinality data DB-tables as we can see in the file 'datastructure' described below. The other tables in the context of 'Controlfile' do not need this kind of statement because they do not have reference to any other table.

\subsection{2 - The Data Structure File for 2DPHOT - UKIDSS}

As in SPIDER case, the file 'datastructure' defines the DB-tables and sets where and how to retrieve input data and to prepare them to be stored in database. As an illustration we show this file in the Fig. 7. In the figure, we can identify through the keywords all the DB-table names described in Sec. 2.2.2. Each keyword (preceeded by a comment line defined by the sign '---') is followed by the field specifications that defines it. First comes the mandatory field 'reference:', which determines the join(s) with related entities. Among the joins we can see the definition: 'processing=<idprocessing>'. Recall Section 4.2.1

\footnotetext{
${ }^{7}$ All ASCII data files are listed in the Controlfile in the field 'file' of keyword 'database'. They are assumed to have to suffix '. dat'
} 


\section{ControlFile}

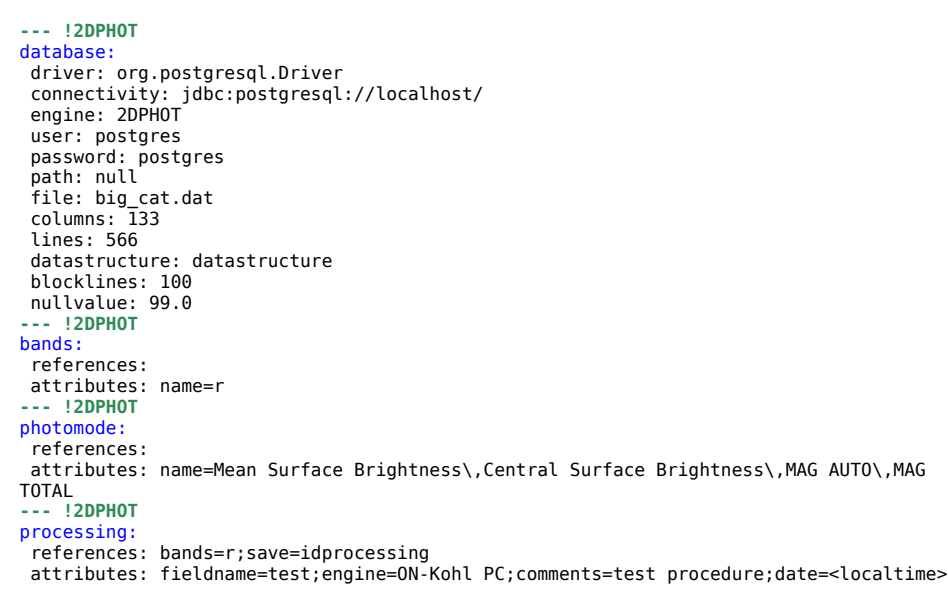

\section{DataStructure}

\section{-.. !2DPHOT}

sexcat:

reference: processing=<idprocessing $>$

idsexcat: indata=big cat.dat; colnumber $=1$; colname $=$ ID

obj class: indata $=$ big cat , dat $;$ colnumber $=2 ;$ colname $=2$ DFLAG

stellarity_idx: indata $=$ big_cat.dat; $\operatorname{colnumber}=3 ;$ colname $=S / G$

photo_flag: indata=big_cat. dat; colnumber $r=4$; colname $=F L A G$

$\mathrm{X}$ : indata=big_cat.dat; colnumber $=5$; colname $=$ XIMA

$y$ : indata $=$ big cat . dat; colnumber $=6$; colname $=$ YIMA

kron radius: indata $=\mathrm{big}$ cat . dat ; colnumber $=7$; colname $=K R A D$

fwhm: indata=big cat . dat ; colnumber $=8$; colname $=F W H M$

isoarea: indata $=\bar{b}$ ig cat . dat ; colnumber $=9$; colname $=A$ REA

$\mathrm{ra}$ : indata=big cat. dat; colnumber $=14 ;$ colname $=R A$

dec_: indata=big_cat. dat; colnumber $=15$; colname $=D E C$

-.. 12DPHOT

photodata:

reference: photomethods=S-extractor; processing=<idprocessing $>$

idsexcat: indata=big cat .dat; colnumber $=1 ;$ colname $=$ ID

ellipticity: indata=big cat .dat; $c$ lnumber $=10$; colname=ELLIP

pa: indata=big_cat.dat; colnumber $=11$; colname $=$ THETA

-.. !2DPHOT

photodata:

reference: photomethods=2DFIT; processing=<idprocessing $>$

idsexcat: indata=big cat. dat; colnumber $=1$; colname $=I D$

ellipticity: indata=big cat .dat; colnumber $=72$; colname=b/a2D; value=\&subtract (1), \$ellipticity. colvalue )

pa: indata=big cat.dat; colnumber $=73$; colname $=P A 2 D$

sersicindex: indata $=$ big cat . dat; colnumber $=74 ;$ colname $=n-2 D$

rms: indata=big_cat.dat; colnumber $=75$; colname $=\wedge$ chi

effectiveradius: indata=big_cat.dat; colnumber $=71$; colname=r_e $\backslash, 2 \mathrm{D}$

$\dot{1}$

Figure 7: Sample of the file 'datastructure' for the database 2DPHOT-UKIDSS.

above describing ControlFile where we mentioned the statement 'save=idprocessing' that should 'save' the primary key of table processing to be retrieved for tables of data. Doing so, the program gets the value of the attribute 'idprocessing' (which is an self-incremented numerical quantity) from database and replaces the keyword <idprocessing> by it.
The other fields, if applied, are defined in the usual way, namely, the statement 'photomethods=GROWTH' means that it should relate to the defined instance of the DB-table (photodata, in this example) to the primary key of the DB-table 'photomethods' having the value 'GROWTH'.
It follows the field specifications for the quantities that DB-tables have. The attribute 'idsexcat' is present in all of them. Because there is only one ASCII table of data, 'big_cat.dat', this at- tribute must be defined for all tables with the same specification, meaning that the input file is 'big_cat.dat', the column is one and its name is 'ID' ${ }^{8}$. The other attributes are defined similarly to

\footnotetext{
${ }^{8}$ Notice first line in the file 'big_cat.dat' in 2DPHOT distribution file.
} 
SPIDER 'datastructure', described in Sec. 4.1.2.

5 - Database Views: The Way for retrieving the Data Tables as Conceived by the Astronomer

As we have seen in Section 1 there may be a huge gap between the way an astronomer sees a database structure and the way the database designer must look at it. View is a tool left available by DBMS vendors for the engineers to design, among other things, relations to look like the tables that users recognize. Astronomers may deal with these relations as it were database tables. Syntactically there is no difference between them. Thereby, whereas an astronomer feels that it is as querying his own tables, though his point of view diverges from the way the database was conceived, there is no threat to the integrity of information. After the views are installed, the user will be able to use these databases with an appropriate application with no further difficulties.

\section{1 - VIEWS in SPIDER}

We introduced fifteen views in database SPIDER covering the quantities originally designated to compose data. They are organized by datatypes mimicking the structure presented on Section 2.1.1. They all have the column idSDSS, aliased as sdss, for they are all related to astronomical objects. ID and photometric data also have the column idbands. Spectroscopic data have idspect. Here are the Views and their quantities (sdss absent):

- ID/positioning data type:

equatorials: ra, dec, epoch;

frame_feat: $x, y$, seeing, scale, sep, a_extinction;

idents: ids, id1, id2.

- Photometric/morphological data type ${ }^{9}$ :

photo_feat: mi_e, re_e, ba, pa, sn;

kcs: std_redshift, Kron, Total, Kron k3 band $i$, Kron $k 4$ band i;

ems: emk, emk3, emk4, emk5, emk6;

mags: magk, magt, magk3, magk4, magk5, magk6;

ebvs: ebv, ebvc;

a4: a4_g, a4_r, a4_i, a4_z, a4_gri, err_a4; var: C(lre,lre), C(mie,mie), C(lre,mie), $\mathrm{C}(\ln , \ln ), \mathrm{C}($ lre, ln), C(mie, ln);

chi2: chi22d, chi2psf.

- Spectroscopic data type:

spec_feat: age, $[\mathrm{Z} / \mathrm{H}],[\mathrm{al} p h a / \mathrm{Fe}]$.

- Cosmological data type:

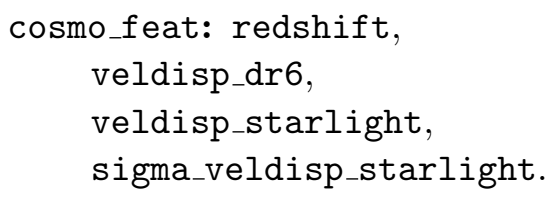

According to the presented in Section 2.1, their names are self explainable.

The entities and attributes shown above are acknowledged by the user and he(she) may apply their queries by joining tables, by choosing bands and other applicable qualifiers at his(her) will. For instance, a very straightforward query may be applied to retrieve the surface brightness inside the effective radius and the total magnitude, both, on the band ' $r$ ':

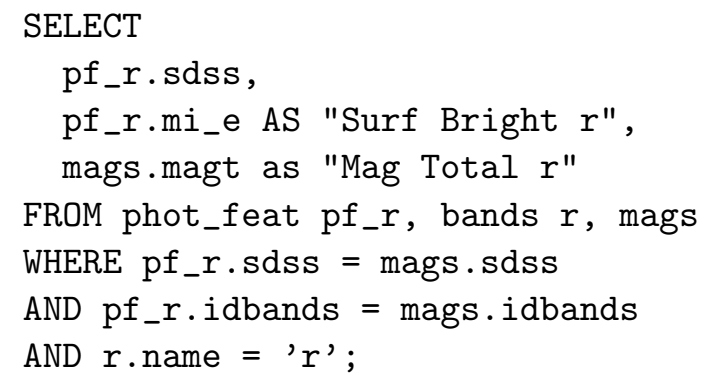

More complex combinations, conditions and other SQL facilities may be applied, too.

\section{2 - Views in 2DPHOT-UKIDSS}

Views were setup in database following the structure showed in Section 2.2.1. As in the SPIDER case, these views may be joined to each other to produce data as presented in ASCII-input file 'big_cat.dat', explained in the doc-files of 2DPHOT distribution. They are:

identification: features in the id/positioning group of data,

id_processing: a uniq identification in database, composed by the sexcat 'id' and the processing table id;

xima, yima: frame coordinates of object;

ra, dec: equatorial coordinates of object;

\footnotetext{
${ }^{9}$ The views ebvs and $\mathbf{a} 4$ have no column idbands.
} 
fwhm: full width half maximum of the object image.

photometry: features of photometric data,

krad: Kron radius;

mauto \& ermauto: 'auto'-magnitude and its error estimator, according to S-extractor user's guide;

mapn \& ermapn: magnitudes and their error estimators for apertures n from 1 to 27 , according to S-extractor user's guide;

$<$ mi >e: surface brightness inside effective radius;

mt: total magnitude.

classification/morphological: all the features concerning the type classification of the object and its morphological information,

2dflag: object class;

stellarity_idx: stellarity index;

photo_flat: photometric flag ${ }^{10}$.

All these last features are linked to a photometric band whose filter was applied to observe the frame the objects come from, and whose identification may be retrieved from the processing.

\section{3 - Querying Matched Fields}

As long as retrieving raw information is concerned, setting up queries from views in 2DPHOTUKIDSS database is relatively simple. Nevertheless, to make science the astronomer must find matched objects from different frames obtained with different (or not) photometric filters. It means that one must somehow populate the DB-tables designed to house the outcomes from the matching applications the astronomer occasionally does (in Fig. 4, the tables inside the lower-left box named "Matching"). Obviously, only whom is granted rights to insert and update these tables is able to store data in these entities. It is up to the DB-administrator to grant whomever is concerned. Once this step is taken, the user may either run a matching algorithm outside database, using its own matching program, or do it by calling an extended function inscribed in the database, or even apply a SQL statement to query and insert/update data into the "Matching-tables".

\footnotetext{
${ }^{10}$ All these quantities come from S-Extractor calculations.
}

Below, we can see a sample of a SQL statement on querying 2DPHOT-UKIDSS database for object positions when they match inside a circle of $1^{\prime}$ diameter:

SELECT i1.id_processing, i2.id_processing, i1.ra as $\mathrm{r} 1$, i1.dec as $\mathrm{d} 1$, i2.ra as $r 2$, i2.dec as d2

FROM identification i1, identification i2 WHERE i1.id_processing != i2.id_processing AND ((i1.ra-i2.ra)*cos (radians (i1.dec)) )^2 $+(i 1 . \operatorname{dec}-i 2 . \operatorname{dec})^{\wedge} 2<2.8 \mathrm{E}-04$

The last condition determines the radius of the circle, which value, in radians, equals $1^{\prime}$. Notice that the right ascension component is multiplied by the cosine of the declination to perform a sort of field planification, which is true except for a position very near the poles. We should set more restrictive constraints in the search and make the query more complex by adding more features from other views, if we want it. Care should be taken to properly join them in order to garantee data integrity. Constraints on photometric band and on specific regions on the sky are likely welcome. It is important to state that the column id_processing is to be different for objects in a tuple in order to avoid matches on the same object.

We can also define a function to be nested inside the database, in terms close to the query showed above, so that we could only parse some parameters, like the radius of matching circle, the region in the sky and the photometric bands involved.

Other way of doing matching process is to write a program in some classic language, like $\mathrm{C}$ or $\mathrm{FOR}$ TRAN, and, by means of some driver that is put available by the DBMS vendor, to connect the database and parse the necessary constraints which the program was designed to provide. This is just a slightly more sophisticated procedure than to dump part of database into the disk and doing the matching on them.

\section{6 - Conclusion}

A permanent database loading program has been developed to facilitate the task of storing astronomical data in a structured database. Two cases very similar in structure but quite universal in presentation are introduced by way of example. A pipeline should be introduced to the concerned astronomers to safely store their data so that they may be retrieved by others or even by themselves. Here, some rules to 
build a type of configuration files lead the program to get data from some ASCII tables, provided these data present a structured pattern, and to save them in a database. Any kind of data, as long as it is in ASCII format (other codification systems depend on the way the DBMS was formated) may be applied.

Structuring database still depends on the designer skills, who depends on 'interviews' with the astronomer to properly understand what all the data means. Obviously this task may be done by the astronomer him(her)self if he(she) has the proper knowledge.

Database may be of fixed or variable cardinality. This condition depends on the way the database was designed and the contents of ASCII files designated to provide newdata. Specific schematic files should be easily produced so the data is stored into the database.

Astronomers may update data or include new ones at will, since possible changes in database structure are communicated to the database administrator. In our experience, astronomers can easily deal with the concept of schematic files we introduce here.

Any database structure may be introduced to this scheme. A strict connection between how data are spanned through ASCII tables and files containing

\section{References}

[1] Hawkins, J.L., 1991, dSTORY; how I really developed dBASE, Data Based Advisor 9:3

[2] Rather, E., Colburn, D.R., Moore, C.H., 1993, The Evolution of FORTH, ACM SIGPLAN Notices $28: 3$

[3] Suad, A. 1986, Relational Database Technology, Springer-Verlag

[4] Hanish, R.J., De Young, D., 2007, Prologue: The Virtual Observatory - A New Environment for Astronomical Research, In GRAHAM, MJ, FITZPATRICK, MJ and MCGLYNN, TA, editors, Astronomical Society of the Pacific Conference Series, 382: 1.

[5] De Carvalho, R.R., Gal, R.R., De Campos Velho, H.F., Capelato H.V., L.A. Barbera, F., Vasconcelos, E.C., Ruiz, R.S.R., Kohl-Moreira, J.L., Lopes, P.A.A., Soares-Santos, M., 2010, The Brazilian Virtual Observatory - a new paradigm for astronomy, J. Comp. Int. Sci., 1:187-206. DOI:10.6062/jcis.2010.01.03.0022. the statements for transfer the data to the database should be setup. Astronomers should mount data by beginning the ASCII tables with a header, on a single line, containing the column names of the table separated by blank spaces. Rules for composing column names may be applied. Data qualification like photometric bands or analysis name of the method, or acronysms may be presented on the column names and it is preferable that one follows rules for composing them. For instance, the photometric band name always goes to the end or beginning of the word. The more coherent the composition of the column names, the easier the statements programming procedure of the schematic files.

Schematic files play an important role in the process. Astronomers with some experience in program designs, no matter the language they use to employ, may easily understand the logic that is behind the lexical of these files.

With the aid of database views properly defined, the astronomer may retrieve data either for rebuilding his data as formerly presented, or for retrieving them in whatever format he wants. He may be sure of data integrity, that is to say, no information loss or be sure of the information he retrieves is effectively related to the object in question.

[6] Tuomi, I., 2002, The Lives and Death of Moore's Law, First Monday 7:11, ISSN 13960466. URL:

http://firstmonday.org/ojs/index.php/fm/article/view/1000.

[7] Kaiser, N., 2009, Moore's Law takes the universe, New astronomy with giga-pixel imagers and peta-byte data archives, In Aerospace conference, 2009 IEEE: 1-2.

DOI:10.1109/AERO.2009.4839301.

[8] Worsley, J.C. , Drake, JD, 2002, Practical PostgreSQL, O'Reilly Media Inc.

[9] Barthes, R., 1977, Elements of Semiology, Hill and Wang.

[10] Sumathi, S. \& Esakkirajan, S., 2007, Fundamentals of Relational Database Management Systems, Springer-Verlag.

[11] La Barbera, F., De Carvalho, R.R., De La Rosa, I.G., Lopes, P.A.A., Kohl-Moreira, J.L., Capelato, H.V., 2010, SPIDER - I. Sample and galaxy parameteres in the grizYJHK wavebands, MNRAS 408:1313-1334.

DOI: 10.1111/j.1365-2966.2010.16850.x. 
[12] La Barbera, F., De Carvalho R.R., KohlMoreira, J.L., Gal, R.R., Soares-Santos, M., Capaccioli, M, Santos, R., Sant'Ana, N., 2008, 2DPHOT: A Multi-Purpose Environment for the Two-Dimensional Analysis of Wide-Field Images, PASP 120:681-702. DOI: 10.1086/588614.

[13] Bagui, S. \& Earp, R., 2012, Database Design Using Entity-Relationship Diagrams, 2nd ed., Taylor \& Francis Group.

[14] La Barbera, F., De Carvalho R.R., De La Rosa, I.G., Gal, R.R., Swindle, R., Lopes, P.A.A., 2010, SPIDER. I.V. Optical and Near-infrared Color Gradients in Early-type Galaxies: New Insight into Correlations with Galaxy Properties, AJ 140: 1528-1556.

DOI:10.1088/0004-6256/140/5/1528.
[15] Frank, L., 1988, Database Theory and Practise, Addison-Wesley Pub. Co.

[16] Bertin, E., Arnouts S., 1996, SExtractor: Software for source extraction, A\&AS 117:393-404. DOI: $10.1051 /$ aas:1996164.

[17] Rohde, D., Drinkwater, M., Gallaher, M., Dows, T., Doyle, M., 2004, Machine Learning for Matching Astronomy Catalogues, In ZHEN RONG, Y, EVERSON, R, HUYUN, Y, editors, Lecture Notes in Computer Science, 3117:702707, Berlin-Heidelberg, Springer-Verlag.

[18] Murtach, F., 1992, A new approach to pointpattern matching, PASP 104:301-307.

DOI: $10.1086 / 132993$. 